\title{
Procesos institucionales en el pago por servicios ambientales, cuenca alta del río Pasto
}

Institutional processes in the payment for ecosystem services, Pasto River Watershed

\author{
Yesenia Alejandra Narváez R $^{1}$; Wilmer Javier Paz A $^{2}$, Mirian del Rosario Guapucal C ${ }^{3}$; \\ Hugo Ferney Leonel ${ }^{4}$.
}

1. Ingeniera Agroforestal, Universidad de Nariño, Pasto, Colombia, nrale.14@gmail.com.

2. Estudiante Ing Agroforestal, Universidad de Nariño, Pasto, Colombia, wjavy@hotmail.com.

3. Docente, I.AF, M.Sc., Universidad de Nariño, Pasto, Colombia, guapucalc@hotmail.com

4. Docente, I.F, Ph.D., Universidad de Nariño, Pasto, Colombia, hleonel@gmail.com.

Citar: NARVAEZ. Y.; PAZ, W.; GUAPUCAL, M.; LEONEL, H. 2016. Evaluación de procesos institucionales en el pago por servicios ambientales, cuenca Alta del río Pasto. Rev. Cienc. Agr. 33(1):64 - 72.

Fecha de recepción: Noviembre 15 de 2015. Fecha de aceptación: Marzo 10 de 2016.

\section{RESUMEN}

El pago por servicios ambientales (PSA), es la disponibilidad que tiene un individuo o grupo social para aprovechar o conservar la oferta ambiental natural. Este estudio se realizó en cuenca alta del río Pasto, donde el principal servicio ambiental, es la provisión hídrica del 85\% de los habitantes de Pasto, Nariño, convirtiéndolo en un ecosistema estratégico que requiere de alternativas viables para su conservación y manejo. El objetivo fue evaluar los procesos relacionados con PSA, adelantados por algunas Instituciones; para ello, se diseñó una entrevista estructurada, aplicada a funcionarios de cuatro instituciones, que contemplaba las variables: institución, conocimiento normativo, recursos financieros, apoyo técnico, actividades realizadas actualmente y posibles alternativas para PSA. Con el programa Decisión Explorer 3.3 y con integrantes de las instituciones se propusieron alternativas para mejorar el PSA. Se pudo determinar que en el corregimiento de Cabrera se están ejecutando las alternativas: implementación de sistemas agroforestales, familias guardabosques y adquisición de predios para avanzar en PSA. Se concluyó que en la Cuenca Alta del río Pasto, el PSA aún es incipiente pero promisorio, hay poco conocimiento institucional y los procesos se han centrado en la compra de tierras para la conservación de fuentes abastecedoras de acueductos rurales y de cabeceras municipales.

Palabras Claves: instituciones, PSA, incentivo, cuenca hidrográfica. 


\begin{abstract}
Payment for ecosystem services (PES) is the opportunity for an individual or social group to gain benefit from or to conserve the natural environmental offer. This study was conducted in the Pasto River Watershed, where the main ecosystem service is providing water supply to $85 \%$ of inhabitants of the city of Pasto, Nariño, making it a strategic ecosystem that requires viable alternatives for its conservation and management. The aim of this study was to evaluate processes related with PES carried out by several institutions, for which we designed a structured interview that was applied to personnel from four institutions and included the following variables: institution, knowledge of the norm, financial resources, technical support, current activities, and possible alternatives for PES. Alternatives for improvement in PES were proposed using the software Decision Explorer 3.3 and with participants from the institutions. We determined that the township of Cabrera is carrying out alternatives such as implementation of agroforestry systems, park ranger families, and acquisition of lands for PES. We concluded that PES in the Pasto River watershed is rudimentary, yet promising; there is little institutional knowledge and processes have focused on buying lands for the conservation of rural and municipal water supply systems.
\end{abstract}

Keywords: institutions, PES, incentive, Watershed.

\section{INTRODUCCIÓN}

Los servicios ambientales son condiciones y procesos por los cuales los ecosistemas naturales y las especies que los constituyen, sostienen y satisfacen las necesidades humanas (Daily, 1997). También se definen como aquellos bienes y servicios que recibe la sociedad, como producto de las funciones ecosistémicas (Costanza et al., 1997). Se asocian desde el punto de vista económico al concepto de externalidades positivas (Kolstad, 2000), donde al ser una transacción voluntaria, de mantenimiento o provisión de un servicio ambiental definido, es reconocida económicamente por al menos un comprador de ese servicio o por lo menos un proveedor del mismo, sólo si el proveedor asegura el servicio ambiental acordado (Wunder, 2006).

A raíz del gran interés suscitado al interior de los países y de las instituciones sobre el pago por servicios ambientales (PSA) como un mecanismo innovador para conservar los recursos naturales y eventualmente diversificar el ingreso rural. En Colombia, el Plan Nacional de Desarrollo (20142018), le asigna al Ministerio de Ambiente, Vivienda y Desarrollo Territorial, "una gestión ambiental y del riesgo que promueva el desarrollo sostenible", dentro del componente relacionado con la biodiversidad, la responsabilidad de desarrollar "un conjunto de instrumentos económicos y financieros que incentiven el conocimiento, incluyendo los mecanismos necesarios para la creación de un sistema de pago por servicios ambientales en Colombia", el cual, se realice a través de proyectos regionales o locales, desarrollados entre particulares en forma voluntaria o también fomentados por las autoridades ambientales y con la concurrencia de recursos públicos y privados (Gómez, 2011).

La cuenca alta del río Pasto es determinante para el desarrollo sustentable del municipio de Pasto y la zona sur del país, su principal virtud son los bienes y servicios ambientales que ofrece a la población presente, abasteciendo el acueducto Centenario que cubre el $85 \%$ de la población de la ciudad de Pasto (N.), aunque estas características no han sido lo suficientemente valoradas y resaltadas, teniendo como consecuencias, una degradación y deterioro de los recursos naturales de la cuenca (GEA, 2008).

La importancia de ésta área en la evaluación de procesos institucionales para PSA se fundamenta en la prioridad que tiene la cuenca en el departamento y en aquellas instituciones encargadas de administrar el mecanismo financiero y de pago por servicios ambientales, los cuales han evidenciado limitados procesos en la cuenca alta del río 
Pasto (GEA, 2008) dado el incumplimiento de la normativa, las mínimas estrategias generadas y la reciente articulación del respectivo programa en las instituciones de la zona.

Por lo tanto, ésta investigación tiene como objetivo, evaluar los procesos institucionales en el pago por servicios ambientales en la Cuenca alta del río Pasto, para promover participativamente una alternativa que contribuya con el mejoramiento de los procesos para PSA.

\section{MATERIALES Y MÉTODOS}

La cuenca alta del río Pasto corresponde al área montañosa limitada en su parte superior por la línea divisoria de agua, asociadas a los ecosistemas paramunos y de bosque alto andino, a una altura superior de $3600 \mathrm{msnm}$, donde permite que la cuenca capte el agua de precipitación y se convierta en agua superficial y va hasta el límite inferior de las microcuencas El Quinche y la quebrada Dolores en el margen izquierdo de la cuenca, zona donde el río entra a la ciudad de Pasto, en la cota $2600 \mathrm{msnm}$; cubre un área de 8000,84ha, correspondiente al 16,58\% del área total de la cuenca. Administrativamente, corresponde al municipio de Pasto, dentro del cual se encuentran cuatro corregimientos: San Fernando, La Laguna, Buesaquillo y Cabrera (GEA, 2008).

La investigación cualitativa de estudio básico o genérico asume una estrategia de investigación principalmente inductiva. El producto de estudio es descriptivo y analítico. El investigador trata simplemente de descubrir y comprender un proceso (Tamayo, 2004). Los datos se obtuvieron a través de entrevistas estructuradas con 15 preguntas, donde las variables a evaluar fueron: instituciones, conocimiento normativo, recursos financieros, apoyo técnico, actividades realizadas actualmente y posibles alternativas para el pago por servicios ambientales en la cuenca alta del río Pasto.
El proceso metodológico, consistió en recopilar información secundaria, realizar una entrevista estructurada a instituciones como Empresa de Obras Sanitarias de Pasto S.A. E.S.P (EMPOPASTO), Corporación Autónoma Regional de Nariño (CORPONARIÑO), Gobernación de Nariño y Alcaldía de Pasto. Luego se analizó la información obtenida, definiendo oportunidades y limitantes, y finalmente, se propuso una alternativa con participación de las instituciones para mejorar el pago por servicios ambientales (Brunett et al., 2010), el cual se analizó con el programa Decision Explorer 3.3, que permitió agrupar y priorizar la alternativa más viable.

\section{RESULTADOS Y DISCUSIÓN}

Actividades de las instituciones para PSA en la cuenca alta del río Pasto. En los territorios que hacen parte de la cuenca alta del río Pasto, se están llevando a cabo PSA en el corregimiento de Cabrera, por medio de instituciones como EMPOPASTO y Alcaldía de Pasto, mediante la implementación de sistemas agroforestales que viabilizan la restauración y conservación del ecosistema que provee el servicio de regulación hídrica; este pago que no es directamente económico, se justifica en especie mediante asistencia técnica e implementación de árboles que contribuyen a captar agua para mantener la oferta hídrica disponible a favor de la comunidad, pero también se busca generar otros servicios como: captura de carbono, incremento de la biodiversidad, control de erosión y sedimentos que permiten mantener o mejorar la calidad de vida y el desarrollo de actividades productivas.

Estas iniciativas han sido dirigidas por EMPOPAST0, lo cual incluye adquisición de predios en la microcuenca Barbero con una área de 47,27ha dedicadas a la conservación, así como también ha desarrollado un programa denominado familias guardabosques con la participación de 30 familias del corregimiento de Cabrera. Las personas poseedoras de territorios cercanos a bosques, se comprometen a no talar y no ampliar la frontera agropecuaria, mediante la firma de un contrato. 
En Colombia, se ha desarrollado un sistema de PSA similar al ejecutado por EMPOPASTO y la Alcaldía, en el cual, el centro para la Investigación en Sistemas Sostenibles de Producción Agropecuaria - (CIPAV), organización no gubernamental para la promoción de sistemas alternativos de producción agropecuaria, ejecutó un proyecto, con el objetivo de incentivar cambios en las actividades ganaderas para mejorar su desempeño ambiental en los temas de biodiversidad y almacenamiento de carbono. La estrategia utilizada por el proyecto es la investigación de sistemas silvopastoriles, la asistencia técnica y PSA mediante el desembolso por la introducción arbórea, facilitando dos servicios: incremento en la biodiversidad y la captura de carbono. Este proyecto se monitorea anualmente y se certifica a los compradores para la realización del pago (Banco Mundial, 2001).

Otro de los territorios de la cuenca alta del rio Pasto donde se ha avanzado frente a este tema, es la microcuenca las Tiendas perteneciente al corregimiento de la Laguna, en el cual se ha adelantado por parte de la Universidad de Nariño el esquema de PSA, financiado por EMPOPASTO. El objetivo del esquema es promover la gestión del agua e identificar los sistemas productivos de mayor impacto en calidad y cantidad de agua de la microcuenca.

\section{Oportunidades y limitantes del PSA en la cuen-}

ca alta del río Pasto. La mayoría de funcionarios institucionales entrevistados (60\%) no tienen claridad del Decreto 0953 de 2013 y la Ley del 99 de 1993 (MINAMBIENTE, 2013), pero el 90\% sabe que el $1 \%$ del total de ingresos del departamento y municipio, son destinados a la adquisición y mantenimiento de las áreas de importancia estratégica con el objetivo de conservar los recursos hídricos o para financiar esquemas de PSA; lo que representa entre $\$ 400.000 .000$ y $\$ 800.000 .000$ millones de pesos al año para ser invertidos en programas y proyectos para dicho fines, manifestando a la vez que aún es insuficiente para las necesidades que se tiene para un esquema de PSA en el departamento y en especial en la cuenca alta del río Pasto, al ser una de las cuencas prioritarias en generación de servicios ambientales.
En Brasil, las municipalidades de Paraná y Minas, reciben el 5\% del impuesto estatal de ventas para financiar programas de conservación en la parte alta de la cuenca y proteger así las fuentes de agua potable, que conlleva a la conservación de un millón de hectáreas de terreno en el Estado de Paraná y Minas (May et al., 2002), de ahí que, éste programa ha tenido mayor inversión estatal comparada con Colombia y ha logrado mayor conservación de los recursos naturales del territorio.

En Costa Rica se introdujo en la ley, un impuesto hacia los combustibles para financiar procesos de conservación forestal y se estableció el Fondo Nacional de Financiamiento Forestal (FONAFIFO), para recaudar fondos y gestionar el PSA (Pagiola, 2002; Perevochtchikova, 2012). Mayrand y Paquin (2004) plantean que el PSA, muchas veces requiere de recursos externos del tipo de subvenciones y donaciones de organizaciones internacionales, para así, cubrir costos iniciales. Es así como el Fondo para el Medio Ambiente Mundial (FMAM), generó impactos positivo en Costa Rica, al invertir \$EU8 millones en el programa de ecomercados, incluidos \$EU5 millones para pagos por conservación forestal en los Corredores Biológicos Mesoamericanos (Ortiz et al., 2003).

Sin embargo, el apoyo externo significa un impulso positivo a corto plazo, dado que el sistema de PSA, puede generar dependencia a dicho apoyo internacional, como sucedió en Pimampiro - Ecuador; donde el retiro del apoyo de la Fundación Interamericana puso en peligro la existencia del sistema de PSA en la zona (Gutman, 2003).

Por otra parte, se tiene que en las instituciones EMPOPASTO, CORPONARIÑO, Alcaldía de Pasto y Gobernación de Nariño, el 50\% consideran alto su apoyo hacia el PSA; sin embargo, el otro $50 \%$ considera que el apoyo es regular, justificándose en los pocos recursos financieros que se designan y el reciente establecimiento de la normativa en el país; indicando de esta manera que el departamento de Nariño apenas está iniciando su proceso de inclusión y establecimiento de PSA. 
En CORPONARIÑO, se enfatiza en los predios adquiridos para la conservación y recuperación de los ecosistemas; para lo cual, definen las actividades de mantenimiento de acuerdo con la especificidad de los mismos. Por lo general, la adquisición de predios se realiza en áreas de importancia estratégica del recurso hídrico, que abastecen a los acueductos municipales y/o veredales. La conservación en las áreas altas de las cuencas para el mantenimiento de los servicios hídricos, es una estrategia puesta en práctica en varios países de América Latina y el Caribe, entre ellos Brasil, Costa Rica, República Dominicana, Ecuador, Honduras y Panamá. En la mayor parte de los casos, el enfoque preferido es el establecimiento de áreas protegidas, más que la creación de sistemas de PSA para mejorar las prácticas de gestión entre los usuarios. No obstante, los esquemas de PSA con base en cuencas hídricas, son de uso creciente y se han puesto a funcionar en varios países, entre ellos, Estados Unidos, México, Colombia, Ecuador, Costa Rica, Honduras y Brasil (Dudley y Stolton, 2003).

Sin embargo, no hay un conocimiento sobre los procesos llevado, a cabo para pago por servicios ambientales en la cuenca alta de río Pasto, para lo cual, CORPONARIÑO considera que los recursos son muy escasos para realizar estudios, a partir de los cuales se puedan implementar esquemas de PSA, además de la falta de conocimiento en la aplicación de los mismos, imposibilita que los entes territoriales dirijan la atención a PSA.

En contraste con lo anterior, Mayrand y Paquin (2004), mencionan que los esquemas de PSA involucran la creación de nuevos mercados con apoyos legales, fiscales e institucionales, pero existe el riesgo de que los costos de transacción resulten mayores que los potenciales beneficios del sistema; por tal razón, el manejo de los costos de transacción se vuelve una prioridad para los esquemas de PSA.

La gobernación de Nariño, tiene pocos conocimientos sobre procesos de PSA llevados a cabo específicamente en la Cuenca Alta de río Pasto. Aun así, puntualizan que la voluntad política, aprovechamiento y conocimiento de la normativa, son las principales dificultades que se han presentado con relación al PSA. Cabe resaltar, que de las instituciones EMPOPASTO, CORPONARIÑO, Alcaldía de Pasto y gobernación de Nariño, tan solo el 40\% de los funcionarios entrevistados tienen claridad del Decreto 0953 de 2013, lo cual dificulta poner en marcha procesos de PSA en la Cuenca alta del río Pasto.

La Alcaldía del municipio de Pasto, se ha enfocado al igual que CORPONARIÑO en la adquisición de predios, donde han destinado gran parte de los recursos financieros, a procesos para PSA, principalmente en el corregimiento de Cabrera, por medio de asistencia técnica gratuita y presencia constante en los territorios, vinculado a servicios de información y capacitación, que permiten el fortalecimiento de las capacidades de los participantes.

EMPOPAST0, es una de las instituciones que en éste estudio, demuestra una amplia trayectoria en el PSA, especialmente de regulación hídrica. Aquí se evidencia la prioridad hacia la cuenca alta de río Pasto, por el desarrollo de procesos como: alternativas productivas, restauración ecológica, programa de familias guardabosques y asistencia técnica. En el caso de las cuencas hidrográficas, la presencia de ciertas instituciones puede facilitar y fortalecer el establecimiento del PSA; por ejemplo, en el Valle del Cauca, las asociaciones de usuarios de agua funcionan como fundaciones privadas para el financiamiento de actividades de conservación de cuencas, con la coordinación de la empresa hídrica local (FA0, 2002). Lo anterior, muestra que el aprovechamiento de las asociaciones o instituciones ya existentes, puede facilitar mucho el establecimiento de PSA y reducir sus costos de transacción.

Las instituciones cumplen una función esencial en los esquemas de PSA, incluida la investigación científica, la asistencia técnica, la certificación, el manejo de fondos, la mercadotecnia y los vínculos con representantes nacionales e internacionales. Además, puede resultar necesaria la creación de nuevas instituciones, como sucede en El Salvador, donde el proyecto nacional Ecoservicios con apoyo del Banco Mundial y el FMAM, permitió fortalecer instituciones para apoyar la creación de mercados 
locales para los servicios ambientales en áreas prioritarias que involucran a 300 productores agrícolas en una zona de 5,000 hectáreas (Rosa et al., 2004).

Acción para mejorar el PSA en la cuenca alta del río Pasto. Dentro de las alternativas planteadas por las instituciones, para promover el PSA en la cuenca, se pueden destacar entre otras: identificación de metodologías de valoración económica, fomentar la investigación para tener bases sólidas en PSA, articulación de los diferentes actores del proceso para la formulación e implementación de PSA, capacitación sobre aplicación de PSA, mayor inversión y destinación de recursos para la implementación de PSA.

Análisis central. La Tabla 1 muestra las alternativas planteadas por las instituciones en orden descendente, de acuerdo con su grado de centralidad; que hace referencia a la unión entre las ideas respecto a sus co-relaciones existentes, además de su causa y efecto.

A partir del análisis central, se identificaron los siguientes actores: fomentar la investigación para tener bases sólidas en PSA, articulación de los diferentes actores del proceso para la formulación e implementación de PSA, capacitación sobre aplicación de PSA y mayor inversión y destinación de recursos para la implementación de PSA.

Para las acciones mencionadas anteriormente, se presentan las siguientes co-relaciones y relaciones de causa y efecto (Figura 1); para permitir así, abarcar todas las acciones y evidenciar su contribución al generar una alternativa prioritaria para el PSA.
Finalmente, se presenta la alternativa priorizada (Tabla 2), teniendo en cuenta las explicaciones presentadas, generadas de una asociación pertinente entre ellas. Todo esto, de acuerdo a lo planteado por los funcionarios de las instituciones y referente a la experiencia sobre procesos de PSA en la cuenca.

En la experiencia de las diferentes instituciones en PSA, se distingue su grado de intervención en la cuenca, para lo cual, con los resultados presentados, se puede categorizar como alternativa central, la articulación de los diferentes actores relacionados al proceso de PSA, con miras a la formulación e implementación de éste en la cuenca.

De esta manera, fomentar la investigación para tener bases sólidas en PSA, capacitación sobre aplicación de PSA, además de mayor inversión y destinación de recursos para la implementación de PSA, permite establecer dentro las metodologías de valoración económica y modelos (SWAT); una articulación de diferentes actores.

Con lo anterior, las instituciones proponen la articulación de actores como; organizaciones comunitarias, organizaciones no gubernamentales, como la Asociación para el Desarrollo Campesino y la Fundación para la Protección y Recuperación del Medio Ambiente, por consiguiente, algunas de las actividades financiadas por las ONG como, las de compra de predios, reforestación, constitución de reservas y fomento a la agricultura orgánica; pueden tener efectos positivos en términos de la biodiversidad (Blanco, 2005; Borda et al, 2010).

Tabla 1. Alternativas de PSA, planteadas por EMPOPASTO, CORPONARIÑO, Alcaldía municipio de Pasto y Gobernación de Nariño, de acuerdo a su grado de centralidad.

\begin{tabular}{ll}
\hline Condición de Centralidad & Alternativa de PSA (Forma descendente) \\
\hline 5 de 5 conceptos & (6) Mayor inversión y destinación de recursos \\
5 de 5 conceptos & (5) Capacitación sobre aplicación de PSA. \\
5 de 5 conceptos & (4) Articulación de los diferentes actores en la formulación e implementación de PSA. \\
5 de 5 conceptos & (3) Fomentar la investigación para tener bases sólidas en PSA. \\
4 de 5 conceptos & (2) Apoyarse en Modelos SWAT \\
4 de 5 conceptos & (1) Identificar metodologías de valoración económica \\
\hline
\end{tabular}




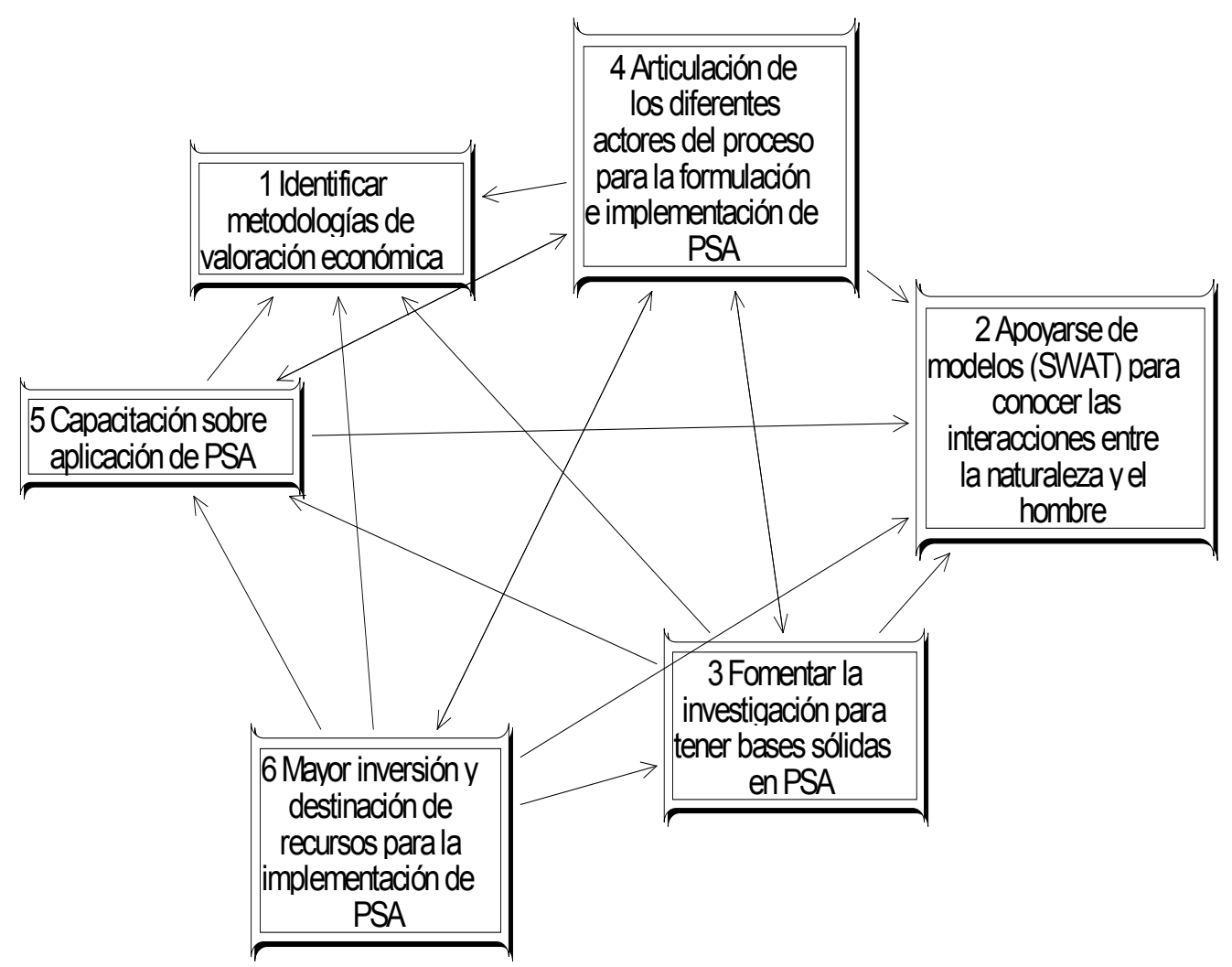

Figura 1. Diagrama de asociaciones más inmediatas de alternativas.

Tabla 2. Alternativa priorizada por medio del análisis de relaciones entre acciones para contribuir al PSA.

\begin{tabular}{ll}
\hline \multicolumn{1}{c}{ Alternativa priorizada } & \multicolumn{1}{c}{ Explicación } \\
\hline & +3 Fomentar la investigación para tener bases sólidas en \\
+4 Articulación de los diferentes actores del & PSA \\
proceso para la formulación e implementación & +5 Capacitación sobre aplicación de PSA \\
de PSA & +6 Mayor inversión y destinación de recursos para la \\
& implementación de PSA \\
\hline
\end{tabular}

También cabe resaltar, la importancia de las asociaciones, como ejemplo en la Cuenca del río Desbaratado en el Valle del Cauca, se logró financiar, entre otras, el plan de manejo de la cuenca, proyectos de educación ambiental y alternativas productivas en la cuenca, como huertos comunitarios y hornillas más eficientes para reducir el consumo de leña (Blanco, 2005) demostrando así, la importancia de las asociación presentes en el sistema de PSA, para la Cuenca Alta de rio Pasto.
Con el apoyo de entidades externas se podría lograr mayor inversión principalmente en esquemas de Pago por Servicios Ambientales, debido a que estos presentan altos costo en las primeras etapas. No obstante, estos esquemas permiten diversos tipos de compensación, que pueden ofrecerse a las comunidades. Además de los pagos monetarios, los esquemas de PSA pueden prever paquetes de compensación con otros beneficios. La experiencia reciente en la materia parece indicar que es preferible apoyar un esquema 
mixto que incluya pagos monetarios y paquetes de compensación con otros beneficios, que van del acceso al crédito al desarrollo de la capacidad y otros servicios colectivos o individuales (Rosa et al., 2004).

En Sarapiquí, Costa Rica, se encontró que los participantes de un sistema PSA en la zona, otorgaron gran valor a la información y entrenamiento recibido en las diferentes capacitaciones, por medio de empresas prestadoras de servicios (Rosa et al., 2004). de ahí, que estos beneficios no monetarios parecen ser altamente valorados por los participantes y son importantes para asegurar la sustentabilidad de los territorios (Halffter, 2011).

Se considera que la articulación permite la unión de esfuerzos de los diferentes actores, quienes han estado trabajando de forma individualizada; sin embargo, han logrado generar cambios dentro de la cuenca alta de rio Pasto, pero se espera proponer mejores alternativas, especialmente en beneficio a los recursos naturales y las comunidades, más aun, siendo la cuenca una de las prioritarias por su virtud en la generación de servicios ambientales.

\section{CONCLUSIONES}

El pago por servicios ambientales (PSA) en la cuenca alta del río Pasto, aún es incipiente pero promisoria, considerando que los funcionarios de las instituciones como alcaldía del municipio de Pasto, CORPONARIÑO, EMPOPASTO y gobernación de Nariño tienen poco conocimiento del PSA, un número reducido de personal e insuficientes recursos para inversión. Los incipientes esfuerzos, se han centrado principalmente en la compra de predios en ecosistemas estratégicos para la conservación de las fuentes abastecedoras de acueductos municipales y veredales. EMPOPAST0 se ha destacado por el desarrollo de procesos como el establecimiento de sistemas agroforestales para captura y almacenamiento de $\mathrm{CO}_{2}$, apoyo a investigaciones para la formulación de un esquema para PSA, consolidación de grupos para la conservación de los recursos naturales asociados al recurso hídrico (guardianes del agua); así mis- mo, CORPONARIÑO, ha establecido un esquema basado en familias Guardabosques.

Las bases centrales para el mejoramiento de los procesos de PSA en la cuenca alta del río Pasto, es la articulación de los diferentes actores en las fases de formulación de estrategias y de ejecución de las mismas, en tanto, se fortalezca la investigación, capacitación, uso de herramientas tecnológicas, mayor inversión e incremento de los recursos financieros y aplicabilidad de la norma.

\section{REFERENCIAS BIBLIOGRAFÍCAS}

BANCO MUNDIAL. 2001. Integrated Silvopastoral Approaches To Ecosystem Management. En: Proyectos y operaciones. http://www.bancomundial.org/projects/P072979/integrated-silvopastoral-approachesecosystem-management?lang=es; consulta: mayo de 2015.

BLANCO, J. 2005. La experiencia de Colombia en esquemas de Pago por Servicios Ambientales. Cifor, Ecoversa. Colombia. 108 p.

BORDA, C.; MORENO, R.; WUNDER, S. 2010. Pagos por Servicios Ambientales en Marcha: La Experiencia en la microcuenca de Chaina, Departamento de Boyacá, Colombia. Primera edición. Centro de Investigación Forestal Internacional (CIFOR), Brasil. 61 p.

BRUNETT, E.; BARÓ, J.; CADENA, E y ESTELLER, M. 2010. Pago por servicios ambientales hidrológicos: caso de estudio Parque Nacional del Nevado de Toluca, México. CIENCIA ergo sum. 17(3): 286 - 294.

COSTANZA, R.; ARGE, R.; GROOTS, R.; FARBER, S.; GRASSO, M.; HANNON, B.; LIMBURG, K.; NAEEM, S.; NELL, R.; PARUELO, J.; RASKIN, R.; SUTTON, P.; BELT, M. 1997. The value of the world's ecosystems services and natural capital. Nature. 387(1):253 - 260.

DAILY, G. 1997. Nature's services societal dependence on natural ecosystems. Primera edición. Washington, Island Press. 98 p.

DUDLEY, N.; STOLTON, S. 2003. Running pure: The importance of forest protected areas to drinking water. Primera edición. WWK. Washington. 114 p. 
FAO. ORGANIZACIÓN DE LAS NACIONES UNIDAS PARA LA ALIMENTACIÓN Y LA AGRICULTURA. 2002. Land-Water Linkages in Rural Watersheds Electronic Workshop -Synthesis Report. En: http//ftp.fao. org/agl/aglw/docs/lw9e.pdf; consulta: junio, 2015.

GÓMEZ. B. E. 2011. Análisis crítico de los Pagos por Servicios Ambientales: de la gestación teorica a la implementación. Revista Española de Estudios Agrosociales y Pesqueros. 228 (1):33 - 54.

GUTMAN, P. 2003. From good-will to payments for environmental services; a survey of financing alternatives for sustainable natural resource management in developing countries. Primera Edición. WWF. Washington. 180p.

GEA. GRUPO DE ESTUDIOS AMBIENTALES. 2008. Plan de ordenamiento y manejo de la cuenca del río Pasto. En: www.pasto.gov.co/.../135-planes-ambientales?... plan-ordenamiento-y-m.; consulta: marzo, 2015.

HALFFTER, G. 2011. Reservas de la Biosfera: Problemas y Oportunidades en México. Acta zoológica mexicana. 21(1):177 - 189.

KOLSTAD, C. 2000. Economía ambiental. Revista Urbana. 7(31):105 - 107.

MAY, P.; VEIGA, F.; DENARDIN, V.; LOUREIRO, W. 2002. Using Fiscal Instruments to Encourage Conservation: Municipal Responses to the 'Ecological' Value-added Tax in Parana and Minas Gerais, Brazil. 173 - 178 pp. En: Pagiola, S., Bishop, J., Landell-Mills, N. Sellinf forest environmental services. Primera edición. Earthscan Publications. Estados Unidos. 180p.

MAYRAND, K PAQUIN, M. 2004. Pago por servicios ambientales. Estudio y evaluación de esquemas vigentes. Primera Edición. Unisfera. Montreal. 65p.

MINAMBIENTE. MINISTERIO DE AMBIENTE Y DESARROLLO SOSTENIBLE. 2013. se reglamenta el artículo 111 de la Ley 99 de 1993 modificado por el artículo 210 de la Ley 1450 de 2011. En: http://wsp.presidencia.gov.co/ Normativa/Decretos/2013/Documents/MAYO/17/DECRET0\%20953\%20DEL\%2017\%20DE\%20MAYO\%20 DE\%202013.pdf; consulta: febrero, 2016.
ORTIZ, E.; SAGE, L.; BORGE, C. 2003. Impacto del programa de pago de servicios ambientales en Costa Rica como medio de reducción de la pobreza en los medios rurales. Primera edición. RUTA, San José, Costa Rica. 65p.

PAGIOLA, S. 2002. Paying for wáter services in Central America: Learning from Costa Rica, pp. 37 - 61. En: Pagiola, S., Bishop, J., Landell-Mills, N. Sellinf forest environmental services. Primera edición. Earthscan Publications. Estados Unidos. 180p.

PEREVOCHTCHIKOVA, M; OCHOA, A. 2012. Avances y limitantes del programa de pago de servicios ambientales hidrológicos en México, 2003-2009. Revista mexicana de ciencias forestales. 3(10):89 - 112.

ROSA, H.; BARRY, D.; KENDEL, S.; DIMAS, L. 2004. Compensation for environmental services and rural communities: lessons from the Americas. Political economy research institute. En: http://scholarworks.umass. edu/cgi/viewcontent.cgi?article=1078\&context=peri_ workingpapers; consulta: mayo, 2015.

TAMAYO, M. 2004. El proceso de la investigación científica. Cuarta edición. Limusa, México D.C. 441 p.

WUNDER, S. 2006. Pagos por servicios ambientales: Principios básicos esenciales. CIFOR Occasional Paper. 42(1):31 - 42. 